\title{
Higher cardiorespiratory fitness predicts long-term survival in patients with heart failure and preserved ejection fraction: the Henry Ford Exercise Testing (FIT) Project
}

\author{
Olusola A. Orimoloye ${ }^{1}$, Swetha Kambhampati², Albert J. Hicks III, Mahmoud Al Rifai ${ }^{1}$, \\ Michael G. Silverman ${ }^{4}$, Seamus Whelton ${ }^{1}$, Waqas Qureshi ${ }^{5}$, Jonathan K. Ehrman ${ }^{6}$, \\ Steven J. Keteyian ${ }^{6}$, Clinton A. Brawner ${ }^{6}$, Zeina Dardari ${ }^{1}$, Mouaz H. Al-Mallah , Michael J. Blaha ${ }^{1}$
}

\begin{abstract}
${ }^{1}$ Ciccarone Center for the Prevention of Cardiovascular Disease, Johns Hopkins University School of Medicine, Baltimore, USA

2Department of Medicine, Johns Hopkins University School of Medicine, Baltimore, MD, USA

${ }^{3}$ Department of Medicine/Cardiology Division, Baylor Scott \& White Health, Temple, USA ${ }^{4}$ Cardiology Division, Massachusetts General Hospital, Boston, MA, USA

${ }^{5}$ Division of Cardiovascular Medicine, Wake Forest University of Medicine, Winston Salem, NC, USA

${ }^{6}$ Division of Cardiovascular Medicine, Henry Ford Hospital, Detroit, MI, USA

${ }^{7}$ King Saud bin Abdulaziz University for Health Sciences, King Abdullah International Medical Research Center, King Abdulaziz Cardiac Center, Ministry of National Guard Health Affairs, Saudi Arabia
\end{abstract}

Submitted: 21 February 2018

Accepted: 11 March 2018

Arch Med Sci 2019; 15, 2: 350-358

DOI: https://doi.org/10.5114/aoms.2019.83290

Copyright @ 2019 Termedia \& Banach

\section{Abstract}

Introduction: Higher cardiorespiratory fitness (CRF) is associated with improved exercise capacity and quality of life in heart failure with preserved ejection fraction (HFpEF), but there are no large studies evaluating the association of HFpEF, CRF, and long-term survival. We therefore aimed to determine the association between CRF and all-cause mortality, in patients with HFpEF. Material and methods: In the Henry Ford Exercise Testing (FIT) Project, 167 patients had baseline HFpEF, defined as a clinical diagnosis of heart failure with ejection fraction $\geq 50 \%$ on echocardiogram. The CRF was estimated from the peak workload (in METs) from a clinician-referred treadmill stress test and categorized as poor (1-4 METs), intermediate (5-6 METs), and moderate-high ( $\geq 7$ METs). Additional analyses assessing the effect of HFpEF and CRF on mortality were also conducted, matching HFpEF patients to nonHFpEF patients using propensity scores.

Results: Mean age was $64 \pm 13$ years, with $55 \%$ women, and $46 \%$ Black. Over a median follow-up of 9.7 (5.2-18.9) years, there were 103 deaths. In fully adjusted models, moderate-high CRF was associated with $63 \%$ lower mortality risk ( $\mathrm{HR}=0.37,95 \% \mathrm{Cl}: 0.18-0.73)$ compared to the poor-CRF group. In the propensity-matched cohort, HFpEF was associated with a HR of 2.3 (95\% Cl: 1.7-3.2) for mortality compared to non-HFpEF patients, which was attenuated to 1.8 (95\% Cl: $1.3-2.5)$ after adjusting for CRF.

Conclusions: Moderate-high CRF in patients with HFpEF is associated with improved survival, and differences in CRF partly explain the intrinsic risk of HFpEF. Randomized trials of interventions aimed at improving CRF in HFpEF are needed.

Key words: cardiovascular disease, risk prediction, risk assessment, heart failure.

\author{
Corresponding author: \\ Dr. Michael J. Blaha \\ Ciccarone Center \\ for the Prevention \\ of Heart Disease \\ Johns Hopkins \\ University School \\ of Medicine \\ Blalock 524D1 \\ 21287 Baltimore, USA \\ Phone: 1-443-287-4960 \\ E-mail: mblaha1@jhmi.edu
}




\section{Introduction}

Heart failure with preserved ejection fraction (HFPEF) accounts for nearly half of heart failure hospitalizations and more than half of the heart failure mortality in the US [1]. A combination of its prevalence, outcomes, and future projections has led to a description of HFpEF as the single largest unmet need in cardiovascular medicine [1]. Despite this high prevalence of HFpEF in the heart failure subpopulation, there are few effective therapies to improve mortality outcomes in these patients and few methods for risk stratification $[2,3]$.

The assessment of cardiorespiratory fitness (CRF), a health-related component of physical fitness defined as the ability of the circulatory and respiratory systems to supply oxygen to skeletal muscles during sustained physical activity [4], has been proposed as a tool for risk stratification in this heart failure subpopulation. There have also been suggestions that improving this measure may lead to better outcomes in patients with HFpEF.

Numerous studies have shown graded inverse associations of morbidity and mortality with CRF across multiple populations [5], including healthy [6], diabetic [7], overweight [8], and hypertensive individuals [9], as well as in patients with heart disease [10], and following myocardial infarction [11]. Similarly, in heart failure with reduced ejection fraction (HFrEF), low CRF has been shown to be associated with increased mortality in patients with ischemic cardiomyopathy, non-ischemic cardiomyopathy, and patients being evaluated for heart transplantation [12-14].

High levels of CRF have been shown to improve or prevent many of the comorbidities commonly seen with HFpEF, including hypertension, obesity, anemia, diabetes, renal dysfunction, and impaired left ventricular compliance [1]. Although these comorbidities have been shown to be associated with HFpEF or the ventricular-vascular dysfunction seen in patients with HFpEF, they do not fully account for the poor outcomes seen in the HFpEF population [15]. When HFpEF patients were compared to matched patients enrolled in clinical trials, the HFpEF population still demonstrated significantly higher mortality [15].

Currently, there are limited data examining the association of CRF and survival in HFpEF patients [16]. Trials of exercise training in HFpEF populations have only reported short-term follow-up data, and are thus not adequately powered to evaluate mortality [17-19]. This lack of large HFpEF cohorts, multiple negative treatment trials, and lack of long-term follow-up have contributed to the paucity of interventions in HFpEF and methods for HFpEF risk stratification. Understanding the association of CRF and mortality in patients with HFpEF is an important step toward developing interventions aimed at prolonging life in patients with HFpEF. Programs such as cardiac rehabilitation may be better supported and reimbursed with data suggesting that such interventions may improve survival in these patients.

Given the foregoing, we aimed to study the association between CRF and long-term mortality in patients with HFpEF isolated from the Henry Ford Exercise Testing Project (the FIT Project) cohort. We hypothesized that higher CRF would be independently associated with lower risk of death and that this association would be similar for both HFpEF and non-HFpEF individuals.

\section{Material and methods}

\section{Study design}

We examined data from the FIT Project, an investigator-initiated retrospective cohort study of 69,885 patients who underwent a physician-referred exercise stress testing at Henry Ford Health System affiliated subsidiaries in metropolitan Detroit, MI between 1991 and 2009. The FIT Project rationale and design have been previously described [20]. Briefly, the FIT Project was assembled using directly measured exercise data, and retrospectively collected medical and medication history. Verification and supplementation of missing data was performed using electronic medical records (EMR), administrative databases, and pharmacy claims files. The FIT Project determined total mortality via linkage with the Social Security Administration Death Master File, and select nonfatal outcomes through medical claims files [20].

\section{Study cohort and identification of HFpEF}

The study sample consists of individuals within the FIT project who had a prior clinical diagnosis of congestive heart failure and available treadmill data, who demonstrated ejection fraction of $\geq 50 \%$ within 1 year of the clinical treadmill test.

The clinical diagnosis of heart failure was made either during the intake report taken by the nurse at the start of the test or by an EMR-based diagnosis requiring at least three occurrences of heart failure ICD-9 code 428.XX in the patient's chart. In all cases, the history of heart failure was confirmed by linkage with administrative claims files confirming a history of a billing diagnosis of heart failure.

Specifically, we first considered 1,596 patients who had documented heart failure within the FIT project. Of this number, 575 had a clinical treadmill test within 1 year of an echocardiogram (the median time from treadmill test to echocardiogram in our HFpEF cohort was 53 days). Of this number, a total of 165 patients had an echocardiogram with a documented ejection fraction (EF) 
$\geq 50 \%$, and these comprise the study population. HFpEF was thus defined in this study as an established clinical diagnosis of heart failure with an associated ejection fraction $(\mathrm{EF}) \geq 50 \%$ on echocardiogram.

\section{Mortality assessment}

The primary outcome was all-cause mortality with a median follow-up time of 9.7 (5.2-13.9) years. Mortality ascertainment was conducted using social security number, first name, last name, and date of birth to search the Social Security Death Index Death Master File until April 2013. A complete search was done in over $99.5 \%$ of all the patients in the FIT database [20].

\section{Treadmill stress testing and estimation of cardiorespiratory fitness}

All patients underwent routine clinical treadmil stress testing using the standard protocol [21]. Indication for stress test referral was provided by the referring physician, and subsequently categorized into common indications (chest pain, shortness of breath, "rule out" ischemia, or other). The treadmill test was symptom-limited and was terminated if the patient had exercise-limiting chest pain, shortness of breath, or other limiting symptoms as assessed by the supervising clinician. In accordance with American Heart Association and American College of Cardiology (AHA/ACC) guidelines [22], testing could be terminated early at the discretion of the clinician for significant arrhythmias, abnormal hemodynamic responses, diagnostic ST-segment changes, or if the participant was unwilling or unable to continue.

Resting heart rates and blood pressures were taken in the seated position prior to stress testing, and percent of maximal heart rate achieved was based on the age-predicted maximal heart rate formula: 220 - age. CRF was expressed in metabolic equivalents of task (METs), which was calculated by the Quinton treadmill controller based on achieved speed and elevation [23]. Full MET credit for a stage was provided once a patient completed at least $1.5 \mathrm{~min}$ of the 3-minute stage. The following CRF categories were used: poor (1-4 METs), intermediate (5-6 METs), and moderate-high ( $\geq 7$ METs). The poor CRF category was defined based on previous systolic heart failure fitness data which showed worse mortality for patients who achieved 4 or less METs [13].

\section{Measurement of covariates}

Demographic information, indication for stress test, risk factor burden, past medical history, and active medication use were obtained by a nurse and/or exercise physiologist immediately prior to the stress test. A smoking history was defined as self-reported history of smoking. Other risk factors, medical history, and medication data were then augmented by a retrospective search of the EMR. A database-verified diagnosis was considered present when the appropriate International Classification of Diseases, $9^{\text {th }}$ Revision (ICD-9) code was present on more than 3 separate encounters within the Henry Ford health system. Hypertension was defined as a prior diagnosis of hypertension, use of anti-hypertensive medications, or a database-verified diagnosis of hypertension. The blood pressure at the time of the test was not used to diagnose hypertension. Diabetes mellitus was defined as a prior diagnosis of diabetes, use of hypoglycemic medications including insulin, or EMR problem list-based diagnosis of diabetes. A history of coronary artery disease (CAD) was defined as prior myocardial infarction, coronary angioplasty, coronary artery bypass surgery, or prior documented obstructive CAD on a prior angiogram. Weight was measured at the time of initial stress testing. Estimated glomerular filtration rates (GFR) were calculated using the Levey Modification of Diet in Renal Disease formula, where $\mathrm{GFR}=175 \times$ standardized $\mathrm{S}_{\text {creatinine }}{ }^{-1.154} \times$ age $^{-0.203} \times$ 1.212 (if Black) $\times 0.742$ (if female) [24].

\section{Statistical analysis}

Baseline characteristics of the study cohort were summarized for each of the 3 CRF groups. Categorical variables were reported as percent frequencies and compared using the $\chi^{2}$ test, while continuous variables were presented as means \pm standard deviation and compared using ANOVA.

Crude incidence rates of all-cause mortality were calculated and reported per 1000 person-years. Kaplan-Meier survival curves for each CRF group were constructed and compared via log rank testing. The proportional hazards assumption was tested and confirmed using complementary log-log plots. Multivariable Cox regression models were used to assess the association between METs and all-cause mortality using the lowest CRF category as the reference (METs $\leq 4$ ). Model 1 was adjusted for demographics of age, sex, and race. Model 2 was further adjusted for modifiable risk factors including hypertension, diabetes mellitus, smoking status, and weight. Model 3 was additionally adjusted for medication use including $\beta$-blockers, angiotensin-converting enzyme inhibitors (ACEI), angiotensin receptor blockers, and medications for chronic lung disease (i.e. inhalers). Model 4 was further adjusted for history of CAD to account for potential ischemia-related limitations in CRF. We also modeled METs continuously and calculated the risk of all-cause mortality per 1 unit increase of METs. 
Additionally, we sought to evaluate the risk of mortality associated with a HFpEF diagnosis when compared to non-HFpEF patients with similar traditional risk factors and similar CRF. To do this, we created a propensity score-matched cohort of non-HFpEF patients drawn from the larger FIT cohort, excluding patients with heart failure with reduced ejection fraction. Propensity score matching was performed using nearest-neighbor matching in a $1: 3$ fashion accounting for 5 variables (the limit of our statistical power): age, sex, race, hypertension and diabetes mellitus. In subsequent survival analyses using this matched cohort, models were adjusted for smoking status, $\beta$-blockers, ACEI, lung disease medications, known CAD, and statins, as these variables remained residually significantly different between the 2 cohorts.

Finally, to study whether there was a differential benefit of CRF in the HFpEF versus non-HFpEF cohort, we fit separate survival models for
CRF and mortality in patients with and without HFpEF to formally test for effect measure modification.

All reported $p$-values were two-sided and a $p$-value $<0.05$ was considered statistically significant. All analyses were performed using Stata version 13.1 (StataCorp, College Station, Texas).

\section{Results}

Baseline characteristics are summarized in Table I. The mean age of the overall HFpEF cohort was $64 \pm 13$ years, $55 \%$ were female, $46 \%$ were Black and 52\% were white. There were 90 (54\%) HFpEF patients in the poor CRF group, 33 (20\%) in the intermediate group, and 44 (26\%) in the moderate-high group. Patients in the lower CRF categories were older, had a lower eGFR, and were more likely to be female and diabetic $(p<0.05)$. 92.9\% of the entire HFpEF cohort had hyper-

Table I. Baseline characteristics of the study population stratified by METs categories

\begin{tabular}{|c|c|c|c|c|c|}
\hline Parameter & $\begin{array}{c}\text { Total popu- } \\
\text { lation } \\
(N=167)\end{array}$ & $\begin{array}{l}\text { Poor CRF } \\
\text { METs } 1-4 \\
(N=90)\end{array}$ & $\begin{array}{c}\text { Intermediate } \\
\text { CRF } \\
\text { METs 5-6 } \\
(N=33)\end{array}$ & $\begin{array}{l}\text { Moderate- } \\
\text { high CRF } \\
\text { METs } \geq 7 \\
(N=44)\end{array}$ & $P$-value \\
\hline \multicolumn{6}{|l|}{ Demographic characteristics: } \\
\hline Age [years] & $63.9 \pm 12.5$ & $67 \pm 10.9$ & $59.1 \pm 15.9$ & $61.0 \pm 11.1$ & 0.002 \\
\hline Gender, $n, \%$ : & & & & & 0.01 \\
\hline Female & $91,54.5$ & $18,54.6$ & $18,54.6$ & $28,63.6$ & \\
\hline Male & $76,45.5$ & $33,36.7$ & $15,45.5$ & $16,36.4$ & \\
\hline Race, $n, \%$ : & & & & & 0.57 \\
\hline White & $86,51.5$ & $45,50.0$ & $14,42.4$ & $27,61.4$ & \\
\hline Black & $77,46.1$ & $43,47.8$ & $18,54.6$ & $16,36.4$ & \\
\hline Others & $4,2.4$ & $2,2.2$ & $1,3.0$ & $1,2.3$ & \\
\hline \multicolumn{6}{|l|}{ Clinical characteristics: } \\
\hline Hypertension, $n$, \% & $155,92.8$ & $86,95.6$ & $31,93.9$ & $38,86.4$ & 0.15 \\
\hline Diabetes, $n, \%$ & $70,41.9$ & $47,52.2$ & $11,33.3$ & $12,27.3$ & 0.01 \\
\hline History of CAD, $n, \%$ & $94,56.3$ & $51,56.7$ & $18,54.6$ & $25,56.8$ & 0.98 \\
\hline Smoking history, $n$, \% & $55,32.9$ & $29,32.2$ & $9,27.3$ & $17,38.6$ & 0.56 \\
\hline Estimated glomerular filtration rate & $66.9 \pm 30.3$ & $60.6 \pm 33.6$ & $75.8 \pm 22.9$ & $74.3 \pm 24.0$ & 0.03 \\
\hline Weight [lbs] & $179.6 \pm 44.3$ & $176.1 \pm 45.3$ & $176.2 \pm 40.8$ & $189.8 \pm 44.1$ & 0.16 \\
\hline \multicolumn{6}{|l|}{ Medication use, $n$, \%: } \\
\hline$\beta$-Blocker use & $67,40.1$ & $31,34.4$ & $18,54.6$ & $18,40.9$ & 0.13 \\
\hline ACEi use & $79,47.3$ & $45,50.0$ & $17,51.5$ & $17,38.6$ & 0.40 \\
\hline ARB use & $3,1.8$ & $1,1.1$ & $1,3.0$ & $1,2.27$ & 0.75 \\
\hline Use of lung disease medications & $30,18.0$ & 18,20 & $3,9.1$ & $9,20.45$ & 0.045 \\
\hline
\end{tabular}

$A C E$ - angiotensin-converting enzyme inhibitors, $A R B$ - angiotensin receptor blockers, CAD - coronary artery disease. Data represent proportions or means \pm standard deviations. 
O.A. Orimoloye, S. Kambhampati, A.J. Hicks III, M. Al Rifai, M.G. Silverman, S. Whelton, W. Qureshi, J.K. Ehrman, S.J. Keteyian, C.A. Brawner, Z. Dardari, M.H. Al-Mallah, M.J. Blaha

Table II. Mortality rates of the study population with HFpEF stratified by METs categories

\begin{tabular}{|lccccc|}
\hline Death & Total, $n, \%$ & 1-4 METs, $n, \%$ & 5-6 METs, $n, \%$ & $\geq 7$ METs, $n, \%$ & $P$-value \\
\hline At 1 year & $10,6.0$ & $7,7.8$ & $3,9.1$ & 0,0 & 0.14 \\
\hline At 2 years & $17,10.2$ & $13,14.4$ & $4,12.1$ & 0,0 & 0.03 \\
\hline At 3 years & $26,15.6$ & $20,22.22$ & $5,15.2$ & $1,2.3$ & 0.01 \\
\hline At 4 years & $31,18.6$ & $25,27.8$ & $5,15.2$ & $1,2.3$ & 0.001 \\
\hline At 5 years & $40,24.0$ & $32,35.6$ & $6,18.2$ & $2,4.6$ & $<0.001$ \\
\hline At 7 years & $59,35.3$ & $41,45.6$ & $13,39.4$ & $5,11.4$ & $<0.001$ \\
\hline At 10 years & $78,46.7$ & $50,55.6$ & $16,48.5$ & $12,27.3$ & 0.008 \\
\hline
\end{tabular}

tension, while usage of medications such as $\beta$-blockers and ACE Inhibitors was $40.1 \%$ and $47.3 \%$ respectively.

There were 103 (62\%) deaths in the entire HFpEF cohort during the follow-up period. After 5 years, $36 \%$ of patients in the poor CRF category, $18 \%$ of those in the intermediate CRF group, and $4.6 \%$ in the moderate to high CRF group were deceased (Table II). Throughout the follow-up, patients in higher fitness groups maintained a survival advantage over those in lower fitness groups (Figure 1).

The crude incidence rate of death decreased from 84 to 37 deaths per 1000 person-years going from poor to moderate-high CRF. In unadjusted models, compared to the poor CRF group, moderate-high CRF was associated with 64\% lower risk of mortality ( $\mathrm{HR}=0.36,95 \% \mathrm{Cl}: 0.19-0.67)$ (Figure 2). The survival advantage in the moderate-high CRF group persisted in the fully adjusted model $(\mathrm{HR}=0.37,95 \% \mathrm{Cl}: 0.18-0.73)$. No significant risk reduction in mortality was seen when the intermediate CRF group was compared to the poor CRF

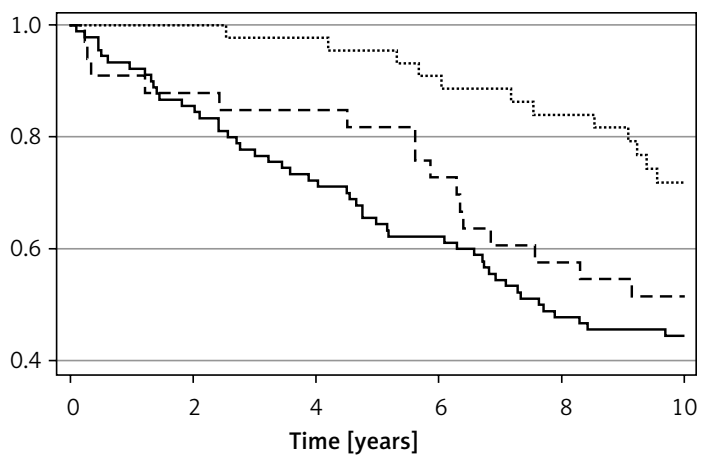

Number at risk:

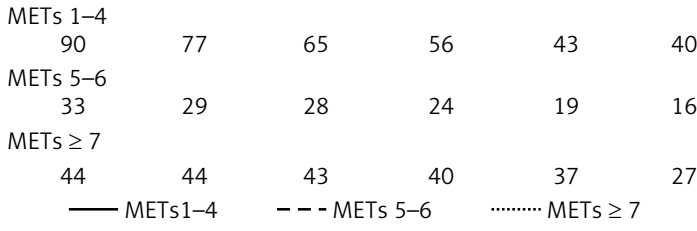

Figure 1. Kaplan-Meier curves of all-cause mortality by METs group for the HFpEF study population group. There was a $13 \%$ reduction in mortality per 1 unit higher METs ( $\mathrm{HR}=0.87,95 \% \mathrm{Cl}$ : 0.78-0.96), which remained significant and unchanged after full adjustment $(\mathrm{HR}=0.86,95 \% \mathrm{Cl}$ : 0.77-0.97).

\section{Risk factor and CRF-adjusted effect of HFpEF on mortality risk}

Baseline characteristics of propensity-matched study population are as described in Table III. After multivariable adjustments, the long-term mortali-

\begin{tabular}{l} 
Multivariable \\
Cox \\
Regression \\
\hline Unadjusted: \\
Reference \\
5-6 METs \\
$\geq 7$ METs \\
Per MET \\
Model 1 (age, sex and race) \\
Reference \\
5-6 METs \\
$\geq 7$ METs \\
Per MET \\
Model 2 (model 1 + risk factors) \\
Reference \\
5-6 METs \\
$\geq 7$ METs \\
Per MET \\
Model 3 \\
(model 2 + risk medications) \\
Reference \\
5-6 METs \\
$\geq 7$ METs \\
Per MET \\
Model 4 \\
(model 3 + history of CAD) \\
Reference \\
5-6 METs \\
$\geq 7$ METs \\
Per MET \\
\hline
\end{tabular}

Figure 2. Multivariable-adjusted hazard ratios (95\% confidence interval) for the association of CRF and all-cause mortality in the HFPEF population 
ty risk of the HFpEF cohort was significantly higher than the propensity-matched non-HFpEF cohort $(\mathrm{HR}=2.3,95 \% \mathrm{Cl}: 1.7-3.2)$. After further adjustment for CRF (modeled as continuous METs), the mortality risk associated with HFpEF diminished but was still significant $(\mathrm{HR}=1.8,95 \% \mathrm{Cl}: 1.3-$ 2.5), suggesting that CRF partly accounted for the association between HFpEF and mortality. Similar results were obtained when we adjusted for METs as a categorical variable (Table IV).

Table III. Baseline characteristics of propensity-matched study population

\begin{tabular}{|c|c|c|c|}
\hline Parameter & Case $(N=167)$ & Control $(N=501)$ & $P$-value \\
\hline Mean METs & $4.7 \pm 2.5$ & $6.3 \pm 2.8$ & $<0.001$ \\
\hline \multicolumn{4}{|l|}{ METs categories, $n$, \%: } \\
\hline $1-4$ & $90,53.9$ & $131,26.2$ & $<0.001$ \\
\hline $5-6$ & $33,19.8$ & $111,22.2$ & \\
\hline$\geq 7$ & $44,26.4$ & $259,51.7$ & \\
\hline \multicolumn{4}{|l|}{ Matched on: } \\
\hline Age [years] & $63.9 \pm 12.5$ & $63.9 \pm 11.3$ & 0.99 \\
\hline Gender, $n, \%$ : & & & 0.89 \\
\hline Females & $91,54.5$ & $270,53.9$ & \\
\hline Males & $76,45.5$ & $231,46.11$ & \\
\hline Race, $n, \%$ : & & & 0.96 \\
\hline White & $86,51.5$ & $264,52.7$ & \\
\hline Black & $77,46.1$ & $226,45.1$ & \\
\hline Other & $4,2.4$ & $11,2.2$ & \\
\hline Diabetes, $n, \%$ & $70,41.9$ & $224,44.7$ & 0.53 \\
\hline Hypertension, $n$, \% & $155,92.9$ & $457,91.22$ & 0.52 \\
\hline \multicolumn{4}{|l|}{ Not matched on: } \\
\hline Smoking, $n, \%$ & $55,32.9$ & $197,39.3$ & 0.14 \\
\hline$\beta$-Blockers, $n, \%$ & $67,40.1$ & $242,48.3$ & 0.07 \\
\hline ACEI, $n, \%$ & $79,47.3$ & $147,29.3$ & $<0.001$ \\
\hline $\mathrm{ARB}, n, \%$ & $3,1.80$ & $7,1.40$ & 0.71 \\
\hline Use of lung disease medications, $n, \%$ & $30,18.0$ & $40,8.0$ & $<0.001$ \\
\hline Known CAD, $n, \%$ & $94,56.3$ & $223,44.5$ & \\
\hline GFR & $66.9 \pm 30.3$ & $77.1 \pm 24.7$ & $<0.001$ \\
\hline Weight & $179.6 \pm 44.3$ & $181.6 \pm 39.4$ & 0.60 \\
\hline Statin, $n, \%$ & $42,25.2$ & $157,31.3$ & 0.13 \\
\hline Death percentage, $n, \%$ & $78,46.7$ & $104,20.8$ & $<0.001$ \\
\hline
\end{tabular}

$A C E I$ - angiotensin-converting enzyme inhibitors, $A R B$ - angiotensin receptor blockers, CAD - coronary artery disease, GFR - glomerular filtration. Data represent proportions or means \pm standard deviations.

Table IV. Association between HFpEF and incident all-cause mortality with and without adjustment for cardiorespiratory fitness

\begin{tabular}{|lll|}
\hline HFpEF & $\begin{array}{c}\text { Unadjusted } \\
\text { HR }(95 \% \mathrm{CI})\end{array}$ & $\begin{array}{c}\text { Adjusted* } \\
\text { HR }(95 \% \mathrm{Cl})\end{array}$ \\
\hline Without METs & $2.6(2.0-3.5)$ & $2.3(1.7-3.2)$ \\
\hline With METs (continuous) & $1.9(1.4-2.6)$ & $1.8(1.3-2.5)$ \\
\hline With METs (categorical) & $2.0(1.5-2.7)$ & $1.9(1.4-2.6)$ \\
\hline
\end{tabular}

*Models adjusted for smoking status, use of $\beta$-blockers, use of ACE inhibitors, use of lung disease medications, known CAD and statin use, as these variables remained residually statistically significant following propensity matching. 
A

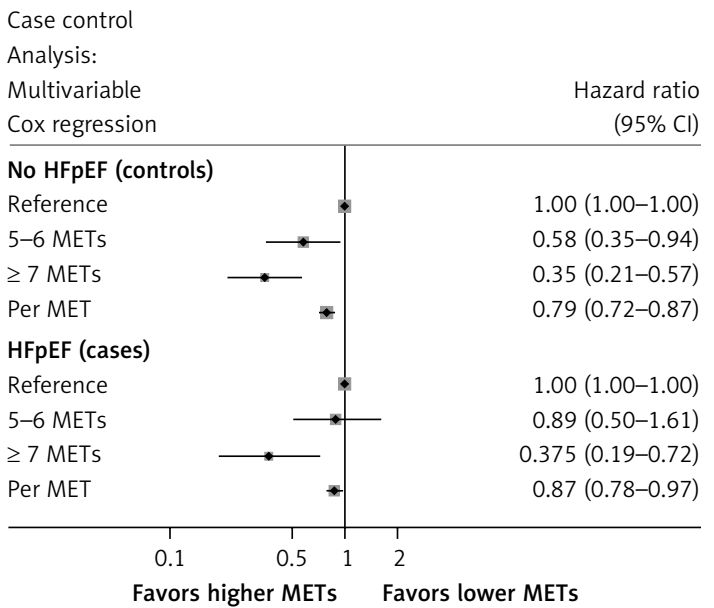

B

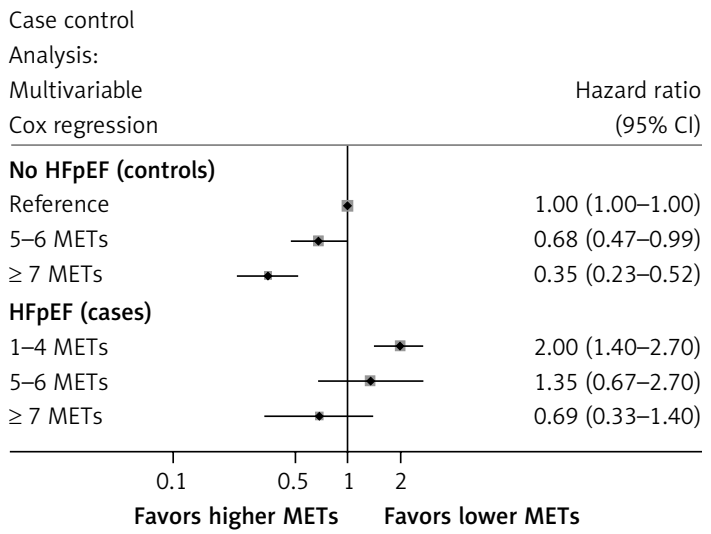

Figure 3. A - Multivariable-adjusted hazard ratios ( $95 \%$ confidence interval) for the association between CRF and incident all-cause mortality by HFpEF status. B - Interaction analysis: multivariable-adjusted hazard ratios (95\% confidence interval) for the association between CRF and all-cause mortality

\section{Assessment of modification of CRF effect by HFpEF status}

The analysis shown in Figure $3 \mathrm{~A}$ presents hazard ratios of mortality with increasing METs for both the HFpEF and non-HFpEF cohorts, with the METs 1-4 category as the reference in each respective group. Higher CRF from poor to moderate-high was significantly associated with lower mortality risk in both the HFpEF and nonHFpEF cohorts, without evidence of interaction $(p=0.32)$, suggesting that the association between CRF and mortality appears similar regardless of HFpEF status.

Figure $3 \mathrm{~B}$ presents hazard ratios of mortality comparing HFpEF and non-HFpEF using the non-HFpEF cohort with poor CRF (METs 1-4) as the reference category. This analysis demonstrated that while moderate-high CRF had a statistically lower mortality risk compared to poor CRF in non-HFpEF patients, HFpEF patients regardless of CRF had similar mortality to the poor CRF nonHFpEF group.

\section{Discussion}

The results of our study demonstrate that in HFpEF patients, higher CRF levels were inversely associated with all-cause mortality compared to poor CRF. We confirm, through a propensity matched analysis, that the diagnosis of HFpEF is independently associated with mortality, and extend existing literature by showing that this risk is partially explained by diminished CRF levels. Furthermore, we found no significant interaction between CRF and HFpEF, suggesting that the improved survival associated with higher CRF appears to be similar for both HFpEF and non-HFpEF cohorts matched for age, gender, and cardiovascular risk factors.
The results of this study are of marked clinical importance as they indicate that data from the straightforward and widely available treadmill exercise stress test can provide important prognostic information in patients with HFpEF, and suggest that improving CRF may potentially yield a mortality benefit in this population.

Our study has several strengths. First, the FIT dataset is the largest epidemiologic database of objectively assessed clinical exercise data to date. Our study population was racially diverse, with nearly $50 \%$ Black subjects, and was also sex-balanced, with nearly $50 \%$ women. In addition, the duration of follow-up extends to 15 years for allcause mortality, with more than $50 \%$ of patients followed for at least 10 years.

In the present study, we showed that HFpEF patients in the FIT Project had a significantly worse mortality per CRF category compared to a propensity-matched cohort, even after multivariable adjustment for other residual differences. We showed however, that this association between HFpEF and mortality is at least partly explained by decreased CRF. This is consistent with other cardiovascular studies that have shown higher mortality rates for HFpEF compared to non-HFpEF patients and suggests that HFpEF is associated with mortality via both CRF-dependent and CRF-independent mechanisms. Studies on HFpEF hemodynamic responses to exercise have described a number of maladaptations that may contribute to the worse outcomes in this population including chronotropic incompetence [25], poor augmentation of cardiac output [26], reduced peripheral arterial blood flow [27], and impaired skeletal muscle metabolism [28]. Indeed, HFpEF patients are known to have poor exercise tolerance demonstrated by a lower peak oxygen uptake, a blunted cardiac 
output increase during exercise, and a robust rise in left ventricular and pulmonary filling pressures compared to healthy non-HFpEF populations [29].

Even though our data suggest improved longterm survival in HFpEF patients who achieved an estimated MET level of 7 or more (moderate-high CRF) on treadmill exercise testing, it is unknown whether targeting exercise training in HFpEF patients to achieve a CRF level of at least 7 METs will improve mortality. Our cohort did show a significant $18 \%$ lower risk for long-term mortality for every MET achieved, so it is possible that improvements in METs through exercise training might show similar long-term mortality benefit in the HFpEF population. Further studies are required to examine whether CRF-oriented exercise training in HFpEF patients will improve survival. Nevertheless, this study demonstrates that the low-cost intervention of a treadmill exercise stress test can provide significant risk stratification for HFpEF patients regardless of multiple comorbidities.

Our results extend the evidence from the findings of prior studies, which have demonstrated that exercise training and improved CRF in patients with HFpEF are associated with clinical improvement. Some studies have also shown that exercise training improves exercise capacity and quality of life, and this benefit is associated with atrial reverse remodeling and improved left ventricular diastolic function through favorable cardiac remodeling [30]. Similarly, a large meta-analysis evaluating the efficacy of exercise training in patients with HFpEF found that exercise training was associated with an improvement in CRF and quality of life [18].

Overall, in the absence of effective pharmacologic treatments available for patients with HFpEF, regular exercise training has shown strong objective evidence in improving quality of life and functional capacity for patients with HFpEF [31, 32]. This study therefore sheds light on improved mortality over long-term follow-up with CRF in patients with HFpEF, which has not been sufficiently demonstrated in the existing literature.

Our study has a number of limitations that warrant acknowledgment. First, the FIT Project reports the experience of a single health system with its unique practice patterns and modes of operation. Although the study population is diverse, it may not be representative of the general US population. Patients who completed a different protocol for stress testing were not included in the FIT Project, potentially excluding patients who are less fit, unable to exercise on a treadmill or those who were referred for pharmacological stress testing. This may have excluded the sickest heart failure patients, given that the protocol used can be challenging for heart failure patients.
Some subjects were allowed to use handrails, so our exercise data may not be fully representative of true exercise capacity. While this may attenuate our effect estimates, this is more representative of what would be obtained in a clinical setting, as it is reflective of clinical safety practices.

In conclusion, moderate-to-high CRF ( $\geq 7$ METs) on baseline treadmill testing is associated with a survival benefit in HFpEF patients. The benefit observed in HFpEF patients is similar to the well-described benefit of CRF in non-HFpEF patients. Future studies should investigate whether targeted exercise training to achieve at least moderate-high CRF would reduce mortality in this high-risk population.

The results of our study demonstrate the importance of measuring CRF in HFpEF patients, with a moderate-high CRF (METs $\geq 7$ ) inversely associated with all-cause mortality after multivariable adjustment. We confirm that a HFpEF diagnosis is independently associated with mortality, and extend existing literature by showing that this risk is partially explained by diminished CRF levels. Furthermore, there appears to be no evidence of interaction between CRF and HFpEF, suggesting that improved survival associated with higher CRF appears to be similar for both HFpEF and non-HFpEF populations matched for age, gender, and cardiovascular risk factors.

Our data alert clinicians to the importance of CRF in determining long-term survival in patients with HFpEF. This study also suggests that strategies to increase CRF, such as cardiac rehabilitation, may potentially be beneficial in the management of patients with HFpEF.

\section{Acknowledgments}

Olusola A. Orimoloye and Swetha Kambhampati are joint first authors.

The authors thank the staff and participants of the FIT Project for their important contributions.

\section{Conflict of interest}

The authors declare no conflict of interest.

\section{References}

1. Butler J, Fonarow GC, Zile MR, et al. Developing therapies for heart failure with preserved ejection fraction: current state and future directions. JACC Heart Fail 2014; 2: 97-112.

2. Cypen J, Ahmad T, Testani JM, DeVore AD. Novel biomarkers for the risk stratification of heart failure with preserved ejection fraction. Curr Heart Fail Rep 2017; 14: 434-43.

3. Pierre-Louis B, Rodriques S, Gorospe V, et al. Clinical factors associated with early readmission among acutely decompensated heart failure patients. Arch Med Sci 2016; 12: 538-45. 
4. Lee D, Artero EG, Xuemei Sui, Blair SN. Review: mortality trends in the general population: the importance of cardiorespiratory fitness. J Psychopharmacol 2010; 24 (4 Suppl): 27-35.

5. Kim J, Al-Mallah M, Juraschek SP, et al. The association of clinical indication for exercise stress testing with allcause mortality: The FIT Project. Arch Med Sci 2016; 12: 303-9.

6. Blair SN, Kohl III HW, Paffenbarger Jr RS, Clark DG, Cooper KH, Gibbons LW. Physical fitness and all-cause mortality. A prospective study of healthy men and women. JAMA 1989; 262: 2395-401.

7. Wei M, Gibbons LW, Kampert JB, Nichaman MZ, Blair SN. Low cardiorespiratory fitness and physical inactivity as predictors of mortality in men with type 2 diabetes. Ann Intern Med 2000; 132: 605-11.

8. Wei M, Kampert JB, Barlow CE, et al. Relationship between low cardiorespiratory fitness and mortality in normal-weight, overweight, and obese men. J Am Med Assoc 1999; 282: 1547-53.

9. Church TS, Kampert JB, Gibbons LW, Barlow CE, Blair SN. Usefulness of cardiorespiratory fitness as a predictor of all-cause and cardiovascular disease mortality in men with systemic hypertension. Am J Cardiol 2001; 88: 651-6.

10. Keteyian SJ, Brawner CA, Savage PD, et al. Peak aerobic capacity predicts prognosis in patients with coronary heart disease. Am Heart J 2008; 156: 292-300.

11. Dutcher JR, Kahn J, Grines C, Franklin B. Comparison of left ventricular ejection fraction and exercise capacity as predictors of two- and five-year mortality following acute myocardial infarction. Am J Cardiol 2007; 99: 436-41.

12. Saxon LA, Stevenson WG, Middlekauff HR, et al. Predicting death from progressive heart failure secondary to ischemic or idiopathic dilated cardiomyopathy. Am J Cardiol 1993; 72: 62-5.

13. Mancini DM, Eisen H, Kussmaul W, Mull R, Edmonds LH, Wilson JR. Value of peak exercise oxygen consumption for optimal timing of cardiac transplantation in ambulatory patients with heart failure. Circulation 1991; 83: 778-86.

14. Keteyian SJ, Patel M, Kraus WE, et al. Variables measured during cardiopulmonary exercise testing as predictors of mortality in chronic systolic heart failure. J Am Coll Cardiol 2016; 67: 780-9.

15. Campbell RT, Jhund PS, Castagno D, Hawkins NM, Petrie MC, McMurray JJ V. What have we learned about patients with heart failure and preserved ejection fraction from DIG-PEF, CHARM-preserved, and I-PRESERVE? J Am Coll Cardiol 2012; 60: 2349-56.

16. Shafig A, Brawner CA, Aldred HA, et al. Prognostic value of cardiopulmonary exercise testing in heart failure with preserved ejection fraction. the Henry Ford HosplTal CardioPulmonary EXercise Testing (FIT-CPX) project. Am Heart J 2016; 174: 167-72.

17. Edelmann F, Gelbrich G, Düngen HD, et al. Exercise training improves exercise capacity and diastolic function in patients with heart failure with preserved ejection fraction. J Am Coll Cardiol 2011; 58: 1780-91.

18. Pandey A, Parashar A, Kumbhani DJ, et al. Exercise training in patients with heart failure and preserved ejection fraction meta-analysis of randomized control trials. Circ Hear Fail 2015; 8: 33-40.

19. Fujimoto N, Prasad A, Hastings J, et al. Cardiovascular effects of 1 year of progressive endurance exercise training in patients with heart failure with preserved ejection fraction. Am Heart J 2012; 164: 869-77.

20. Al-Mallah MH, Keteyian SJ, Brawner CA, Whelton S, Blaha MJ. Rationale and design of the Henry Ford exercise testing project (the FIT project). Clin Cardiol 2014; 37 . 456-61.

21. Bruce RA, Kusumi F, Hosmer D. Maximal oxygen intake and nomographic assessment of functional aerobic impairment in cardiovascular disease. Am Heart J 1973 85: 546-62.

22. Gibbons RJ, Balady GJ, Bricker JT, et al. ACC/AHA 2002 guideline update for exercise testing: summary article: a report of the American College of Cardiology/American Heart Association Task Force on Practice Guidelines (Committee to Update the 1997 Exercise Testing Guidelines). Circulation 2002; 106: 1883-92.

23. Exercise AHACo. Exercise Testing and Training of Apparently Healthy Individuals: A Handbook. American Heart Association. Committee on Exercise - Google Books. American Heart Association; 1972. https://books.google.com/books/about/Exercise_testing_and_training_ of apparen.html?id=901qAAAAMAAJ. Accessed February 11, 2018.

24. Levey AS, Stevens LA, Schmid $\mathrm{CH}$, et al. A new equation to estimate glomerular filtration rate. Ann Intern Med 2009; 150: 604-12.

25. Borlaug BA, Melenovsky V, Russell SD, et al. Impaired chronotropic and vasodilator reserves limit exercise capacity in patients with heart failure and a preserved ejection fraction. Circulation 2006; 114: 2138-47.

26. Borlaug BA, Olson TP, Lam CSP, et al. Global cardiovascular reserve dysfunction in heart failure with preserved ejection fraction. J Am Coll Cardiol 2010; 56: 845-54.

27. Bhella PS, Prasad A, Heinicke K, et al. Abnormal haemodynamic response to exercise in heart failure with preserved ejection fraction. Eur J Heart Fail 2011; 13: 1296-304.

28. Matsue Y, Suzuki M, Nagahori W, et al. Endothelial dysfunction measured by peripheral arterial tonometry predicts prognosis in patients with heart failure with preserved ejection fraction. Int J Cardiol 2013; 168: 36-40.

29. Kitzman DW, Higginbotham MB, Cobb FR, Sheikh KH, Sullivan MJ. Exercise intolerance in patients with heart failure and preserved left ventricular systolic function: failure of the Frank-Starling mechanism. J Am Coll Cardiol 1991; 17: 1065-72.

30. Brinker SK, Pandey A, Ayers CR, et al. Association of cardiorespiratory fitness with left ventricular remodeling and diastolic function: the cooper center longitudinal study. JACC Hear Fail 2014; 2: 238-46.

31. Rogers FJ, Gundala T, Ramos JE, Serajian A. Heart failure with preserved ejection fraction. J Am Osteopath Assoc 2015; 115 : 432.

32. Smolis-Bąk E, Rymuza H, Kazimierska B, et al. Improvement of exercise tolerance in cardiopulmonary testing with sustained safety after regular training in outpatients with systolic heart failure (NYHA III) and an implantable cardioverter-defibrillator. Prospective 18-month randomized study. Arch Med Sci 2017; 13: 1094-101. 